\title{
Very small beam-size measurement by a reflective synchrotron radiation interferometer
}

\author{
T. Naito and T. Mitsuhashi \\ KEK: High Energy Accelerator Research Organization, 1-1 Oho, Tsukuba, Ibaraki 305-0801, Japan
}

(Received 29 August 2006; published 27 December 2006)

\begin{abstract}
A synchrotron radiation (SR) interferometer with Herschelian reflective optics has been developed for the measurement of beams of several $\mu \mathrm{m}$ in size. In a conventional refractive SR interferometer, the dispersion effect of the objective lens limits the instrument to a smaller range of beam-size measurements. To avoid this problem, we designed a Herschelian arrangement of reflective optics for the interferometer. The effectiveness of the reflective SR interferometer was confirmed at the KEK Accelerator Test Facility (ATF) damping ring. The measured vertical beam size obtained using the reflective SR interferometer was $4.7 \mu \mathrm{m}$ and the estimated vertical emittance was $0.97 \times 10^{-11} \mathrm{~m}$.
\end{abstract}

DOI: 10.1103/PhysRevSTAB.9.122802

PACS numbers: 41.60.Ap, 41.85.Ew, 42.25.Hz

\section{INTRODUCTION}

An extremely low-emittance beam is required for the international linear collider to increase the luminosity of high-energy physics experiments [1]. The damping ring (DR) of the KEK Accelerator Test Facility (ATF) was constructed to establish techniques for the production of an extremely low-emittance beam for the linear collider [2]. The operation energy of the DR is $1.3 \mathrm{GeV}$. The designed emittances are $1 \times 10^{-9} \mathrm{~m}$ in the horizontal direction and $1 \times 10^{-11} \mathrm{~m}$ in the vertical direction, with the assumption of $1 \%$ coupling. The expected beam size at one location is $26 \mu \mathrm{m}$ and $5 \mu \mathrm{m}$ in the horizontal and vertical directions, respectively. The beta functions are $\beta x=$ $1.5 \mathrm{~m}$ and $\beta y=2.5 \mathrm{~m}$, respectively, at the location. The measurement technology for such a small emittance is also a development issue.

A synchrotron radiation (SR) interferometer is a useful beam-size monitor to measure beam size in storage rings using the visible light of SR [3]. The resolution of an SR interferometer is smaller than the diffraction limit of imaging by a telescope. SR interferometers have already been applied to several storage rings [3-7]. The SR interferometer is one of the candidates for measuring vertical beam size in the DR on the order of less than $10 \mu \mathrm{m}$. We have already succeeded in measuring a vertical beam size of $14 \mu \mathrm{m}$ in the DR using an SR interferometer [8,9]. Since then, the vertical emittance tuning method of the DR has been improved [10,11], and some of the measurement data have shown evidence of the design emittance. However, the SR interferometer with refractive optics showed a limitation in small beam-size measurements of around $7 \mu \mathrm{m}$. This limitation is mainly due to the dispersion effect of the objective lens.

The SR interferometer uses the quasimonochromatic wavelength of the light to obtain sufficient light intensity. When the wave front of the quasimonochromatic light passes through the objective lens, the dispersion effect appears. The dispersion effect introduces a visibility change, especially in the case of small beam-size measure- ment. This problem cannot be solved by refractive optics using an optical lens. Only reflective optics using a concave mirror can provide a solution. We developed Herschelian reflective optics instead of refractive optics for the SR interferometer. The reflective optics can also use a shorter wavelength, which extends the limit of the measurement. The design of the Herschelian optics and the measurement results are described in this paper. A result of emittance tuning using this monitor is also described.

\section{MEASUREMENT PRINCIPLE AND RESOLUTION}

\section{A. Measurement principle}

The SR interferometer is a wave-front-division-type two-beam interferometer using polarized quasimonochromatic rays. The photons of the SR produce an interferogram after passing through a double slit, filters, and a focusing system. The beam size is estimated from the visibility of the interferogram, which indicates the complex degree of spatial coherence of the photons.

The profile of the object of the light source is given by the Fourier transform of the complex degree of spatial coherence from Van Citert-Zernike's theorem [12]. When a normalized beam distribution, $f(y)$, is assumed, the complex degree of spatial coherence $(\gamma)$ is given by the Fourier transform of the function of the profile $(f)$, as the function of position $y$, as follows:

$$
\gamma(\nu)=\int f(y) \exp (-i 2 \pi \nu y) d y .
$$

The spatial frequency, $\nu$, is

$$
\nu=\frac{D}{\lambda L},
$$

where $\lambda$ denotes the wavelength, $L$ denotes the distance from the light source to the double slit, and $D$ denotes the slit separation. The intensity of the interferogram pattern, $I$, is shown as the function of the position, $y_{0}$, 


$$
I\left(y_{0}\right)=I_{0}\left[\operatorname{sinc}\left(\frac{2 \pi a}{\lambda R} y_{0}\right)\right]\left[1+|\gamma| \cos \left(\frac{2 \pi D}{\lambda R} y_{0}+\varphi\right)\right],
$$

where $a$ denotes half of the slit height of the double slit, $R$ denotes the distance between the interferogram and the back principal point of the objective lens of the interferometer, $\varphi$ denotes the phase of the interferogram, and $\gamma$ is the complex degree of spatial coherence, which is the Fourier transform of the profile. When the source of the object is assumed to have a Gaussian beam profile, $\gamma$ also has a Gaussian distribution. The beam distribution was confirmed by scanning the slit separation. In the case of vertical beam-size measurement using the SR from the horizontal bending magnet, the measured $\gamma$ as a function of the slit separation agreed well with the Gaussian distribution. In the case of horizontal beam-size measurement, the distribution has to take into account the field-depth effect and the intensity imbalance effect between the double slits [3].

The root-mean-square value of $\gamma$ is denoted by $\sigma_{D}$, when the size of the object of the light source, $\sigma_{y}$, is

$$
\sigma_{y}=\frac{\lambda L}{2 \pi \sigma_{D}} .
$$

The $\gamma$ of the fixed slit distance, $D$, is expressed as

$$
\gamma=\exp \left(-\frac{D^{2}}{2 \sigma_{D}^{2}}\right)
$$

Substituting Eq. (5) into Eq. (4), the size of the object of the light source, $\sigma_{y}$, is then calculated as a function of $\gamma$,

$$
\sigma_{y}=\frac{\lambda L}{\pi D} \sqrt{\frac{1}{2} \ln \left(\frac{1}{\gamma}\right)}
$$

\section{B. Resolution due to measurement error}

Figure 1 shows the simulated visibility as a function of the slit separation for each beam size from 5 to $15 \mu \mathrm{m}$ at $\lambda=400 \mathrm{~nm}$. The applicable slit separation is limited by the opening angle of the SR, which is about $5 \mathrm{mrad}$ for $\lambda=$ $400 \mathrm{~nm}$. This opening angle corresponds to a slit separation of $40 \mathrm{~mm}$ at $7 \mathrm{~m}$ downstream from the source point in the case of the KEK-ATF. The beam size of $5 \mu \mathrm{m}$ corresponds to a visibility of 0.9 at $40 \mathrm{~mm}$ slit separation. The statistical error of the measurement, including the noise of the charge-coupled device (CCD), is about $1 \%$. The measurement of $\gamma$ has an indeterminacy of less than $1 \%$. The error transfer from $\Delta \gamma$ to $\Delta \sigma$ is calculated according to the following equation:

$$
\Delta \sigma \propto \frac{1}{\gamma \sqrt{\ln \left(\frac{1}{\gamma}\right)}} \Delta \gamma .
$$

If $\gamma$ has a $1 \%$ error, and the conditions of the KEK-ATF are

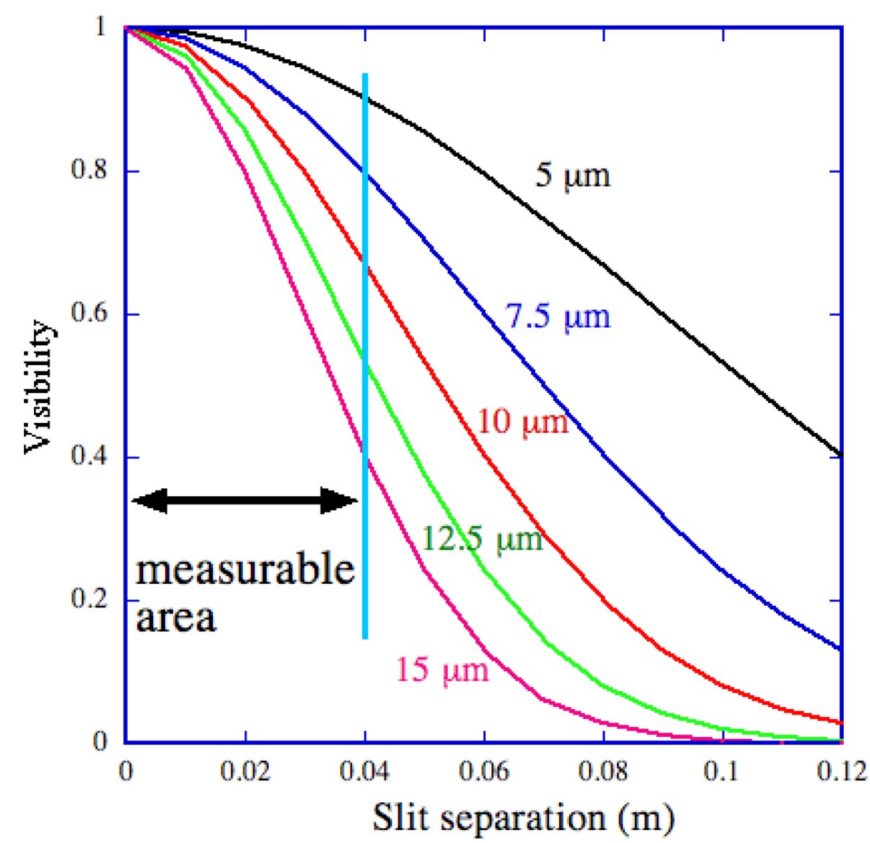

FIG. 1. (Color) Visibility for various beam sizes.

used, the error from $\Delta \gamma$ to $\Delta \sigma$ is as shown in Fig. 2. The measurement resolution by the error transfer is less than $1 \mu \mathrm{m}$ in the region of $\gamma$ from 0.1 to 0.98 . The measurement of the beam size therefore has a $1 \mu \mathrm{m}$ resolution.

\section{Error due to dispersion of the objective lens}

In measuring such good visibility, the most significant error arises from the dispersion effect in the refractive

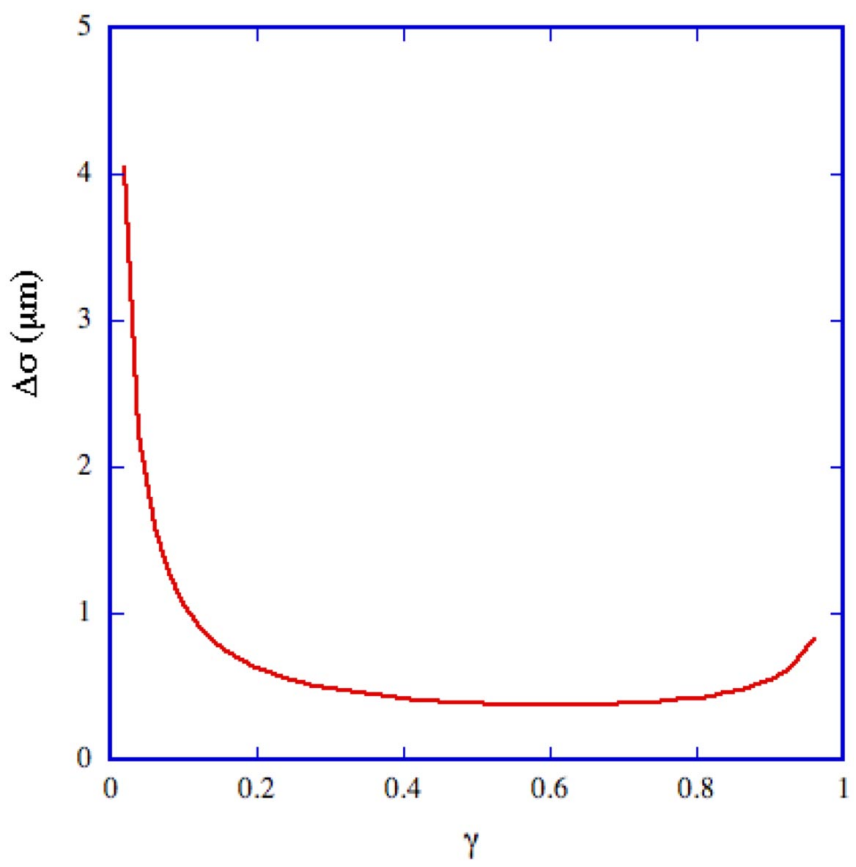

FIG. 2. (Color) Beam size error as a function of $\gamma$, when the error of $\gamma$ is $1 \%$ at each value of $\gamma$. 


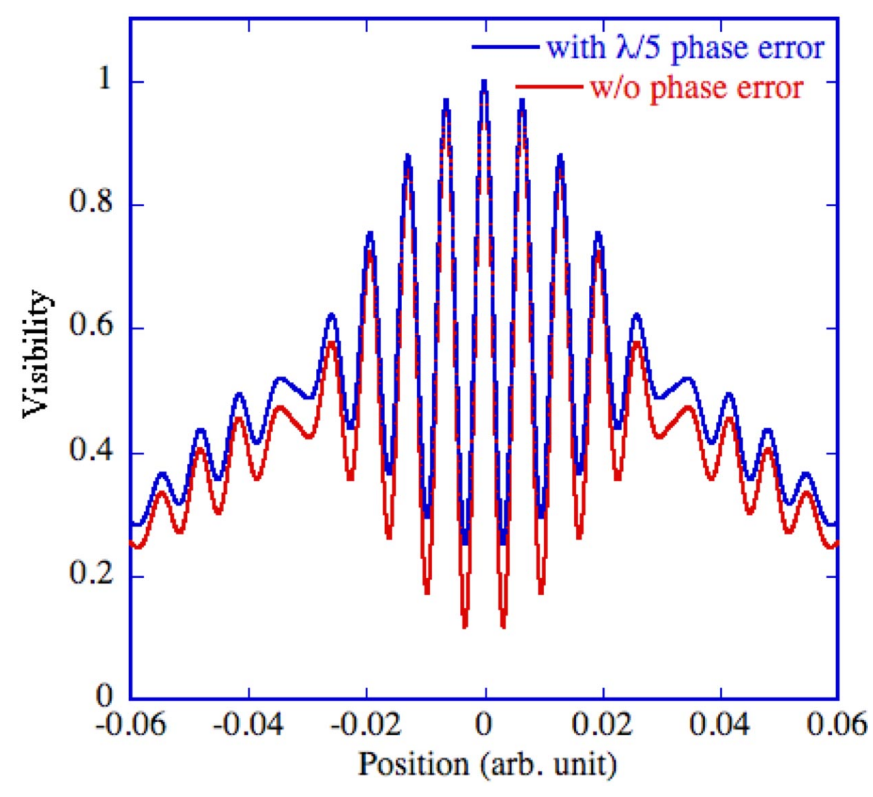

FIG. 3. (Color) Results of interferogram simulations. The green line shows the case of a $5 \mu \mathrm{m}$ beam size without phase error, and the red line shows the case of a $5 \mu \mathrm{m}$ beam size with a phase error of $\lambda / 5$.

optics, particularly if it is from the large aperture of an objective lens having a certain wedge-angle error. In the case of the KEK-ATF, we use a bandpass filter of $80 \mathrm{~nm}$ to obtain sufficient intensity for the interferogram. This wide window of the bandpass filter strongly enhances the effect. The dispersion effect of the lens glass appears as the optical path difference for the different wavelength. This dispersion effect causes a phase change of the interferogram for each wavelength. Consequently, the interferogram will become smeared and the visibility will be reduced. In the conventional SR interferometer having refractive optics, we used an achromatic lens with a focal length of $600 \mathrm{~mm}$ and a diameter of $80 \mathrm{~mm}$ for the objective. The wedgeangle error of this lens is about 0.05 degree at a maximum. Simulated interferograms with and without the dispersion effect are shown in Fig. 3. The visibility of the interferogram is modified from 0.9 to 0.8 when the system has a phase error of $\lambda / 5$.

\section{INTERFEROMETER WITH HERSCHELIAN OPTICS}

Reflective optics was considered in order to avoid the dispersion effect. There are several types of reflective optics for telescope applications. Newtonian optics and Cassegrainian optics are well known as reflective optics. However, the optics layout results in the light at the center of the primary mirror being obstructed by the secondary mirror, and measurement is not possible at a small slit separation. We selected Herschelian reflective optics because it allows simple setting without any obstruction of the SR light and without any aberration in one-dimensional

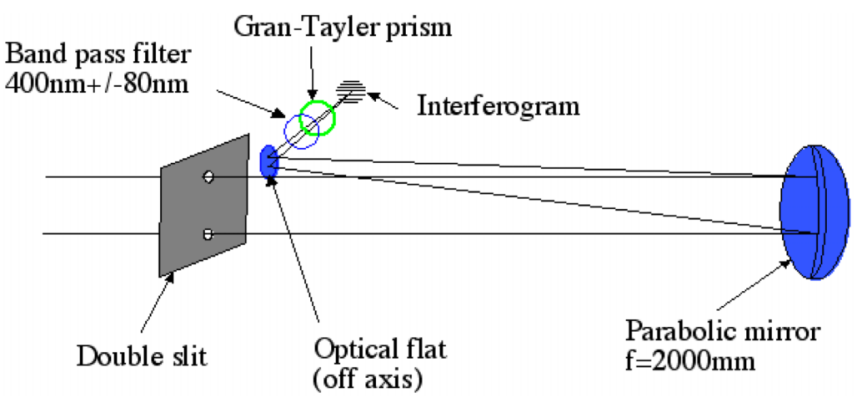

FIG. 4. (Color) Schematic layout of the SR interferometer.

measurement. The reflective optics has no dispersion, and can be used for shorter wavelengths such as $400 \mathrm{~nm}$.

Figure 4 shows a schematic layout of the SR interferometer with Herschelian reflective optics. The double slit is located $7 \mathrm{~m}$ downstream from the source point. A parabolic mirror with a focal length of $2000 \mathrm{~mm}$ is used as the objective mirror. A small diagonal mirror is set for convenience of observation. A bandpass filter having an $80 \mathrm{~nm}$ bandwidth at $400 \mathrm{~nm}$ is used to limit the wavelength of the input light. A Gran-Tayler prism is used to select the $\sigma$-polarization of the SR. To reduce the thermal noise in the CCD camera, we used a cooled CCD camera (Hamamatsu C5985) for observing the interferogram. This CCD has an electric shutter operated by an external trigger. Betatron damping phenomena can be observed with a $1 \mathrm{~ms}$ exposure time by changing the trigger timing. Since mechanical vibration smears the interferogram on the CCD, we set the electric shutter to $1 \mathrm{~ms}$. The setting of a faster exposure time is beneficial for avoiding mechanical vibration, but the SR intensity limits the minimum exposure time in our case. All of the devices are installed on an optical table, and have alignment mechanisms to align the optical path.

\section{EXPERIMENTAL}

The SR is extracted from the bending magnet located at the west arc of the DR. The bending radius is $5.73 \mathrm{~m}$ and the bending field is $1 \mathrm{~T}$. There are four mirrors upstream of the SR interferometer to guide the visible light of the SR. The mirrors have alignment mechanisms to guide the SR. Beam tuning causes a change of the SR orbit, which in turn causes an imbalance in the intensity of the light between the double slits. Careful alignment is needed to avoid any measurement errors arising from an imbalance in the intensity. The imbalance in the intensity must be maintained at less than $\pm 5 \%$ for a visibility error of less than $1 \%$. The slit separation of the double slit is fixed at $40 \mathrm{~mm}$, which is the maximum slit separation used for measurement. The measured CCD image is analyzed by a video analyzer, which consists of a video grabber board and a personal computer. Figure 5 shows an example of an interferogram. The visibility, $\gamma$, is estimated by integrating the light intensity at the region between the vertical lines. 


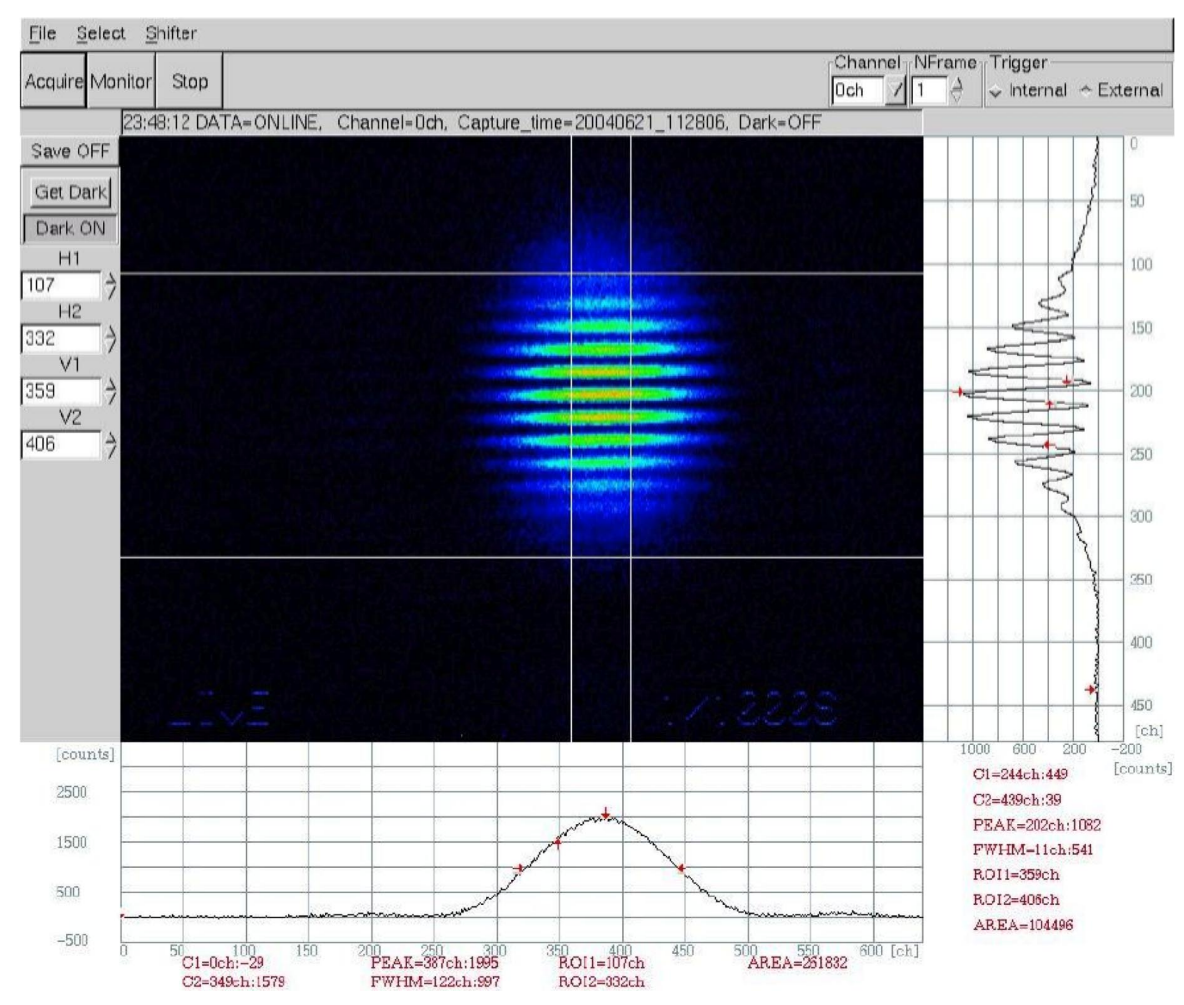

FIG. 5. (Color) Example of an interferogram.

We checked possible measurement error sources other than the dispersion effect of the focus lens, namely, mirror distortion, linearity error of the CCD, and mechanical vibration of the optical components, as follows.

1. Mirror distortion. - There are four mirrors between the source point and the double slit to deliver the SR light. In particular, the first mirror receives the SR power including $\mathrm{x}$ rays and thermal stress, which cause distortion. Mirror distortion changes the effective slit distance in Eq. (6). This effect is very severe in a high-intensity storage ring [4]. We measured the mirror distortion using a pinhole mask. The pinhole mask was set close to the source point of the SR, and its position was moved by a stepping motor. The image of the pinhole was observed at the location of the double slit. The distortion was measured by comparing the moved position of the pinhole and the observed center position of the image of the pinhole at the location of the double slit. The measured mirror distortion was less than $1 \%$ of the effective slit distance when the storage current was $3 \mathrm{~mA}$ under single-bunch operation.

2. Linearity error of the CCD. - The linearity of the CCD was measured by observing constant light, while changing the transmission of the neutral-density filters (NDFs). We used the SR of the KEK Photon Factory as constant light. Figure 6 shows the output of the CCD (Hamamatsu C5985) as a function of the transmission of the NDFs. The measurement exhibited good linearity, even in the low-intensity region. The positive offset at zero intensity can be calibrated by background subtraction at the time of image analysis.

3. Mechanical vibration of the optical components. Mechanical vibration of the optical components causes the interferogram position to move and the visibility to change

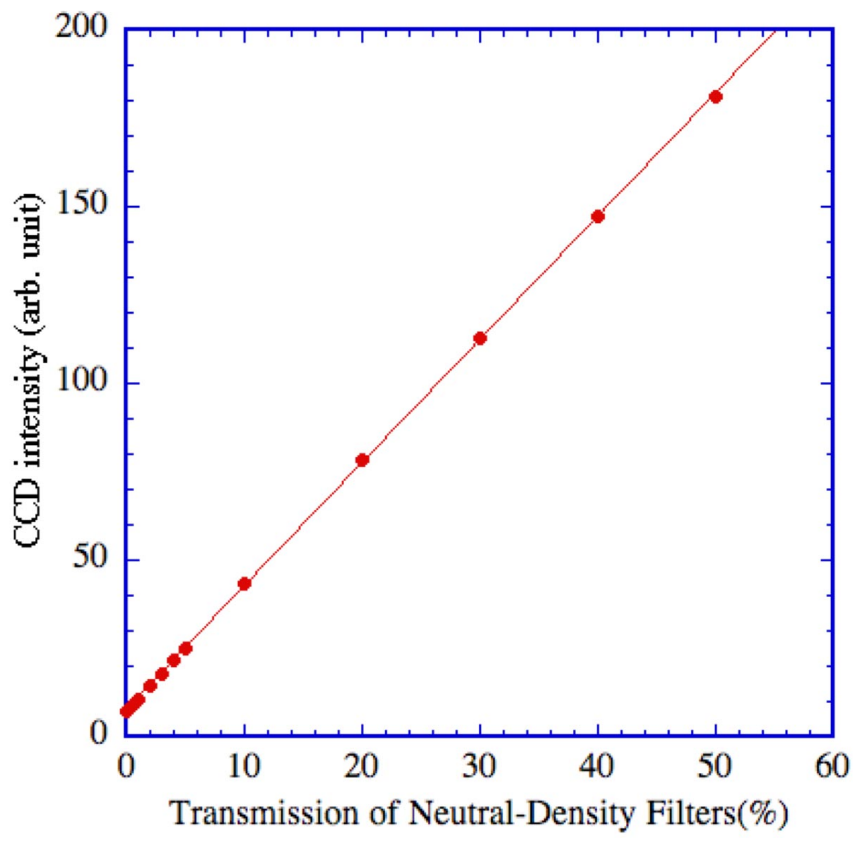

FIG. 6. (Color) Linearity of the CCD output. 


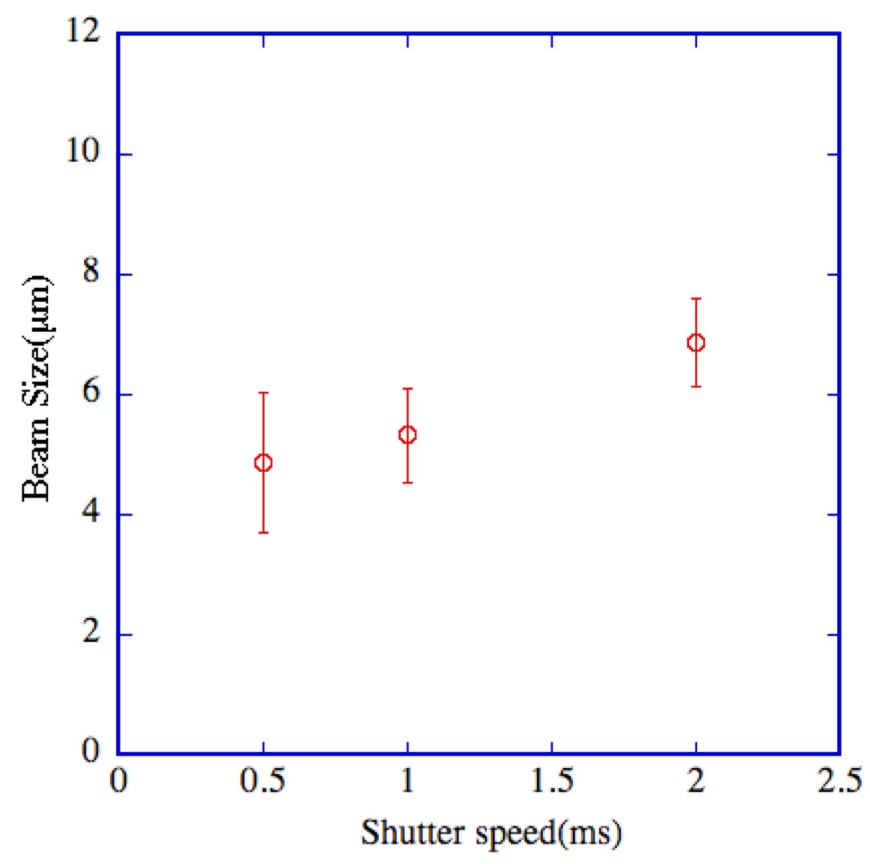

FIG. 7. (Color) Measured beam size as a function of the exposure time.

shot by shot. The effect is very sensitive to the angle of the upstream mirror, particularly in the case of the first mirror. It is very difficult to completely eliminate mechanical vibration because it has many sources; for example, the cooling water of the accelerator magnets and the blowers of the air conditioners. The exposure time of the CCD was reduced so as to avoid the vibration effect. Most of the frequency components of the mechanical vibration exist at lower than $100 \mathrm{~Hz}$. Figure 7 shows the measured beam size as a function of the exposure time. The vibration effect can be reduced at an exposure time of $1 \mathrm{~ms}$ or less. The number of photons during the exposure time limits the minimum exposure time. The signal-to-noise ratio of the CCD increases when the number of photons is small.

\section{MEASUREMENT RESULTS}

The beam size is obtained from the interferogram using Eq. (6). The value of $\gamma$ is evaluated from the measured interferogram and the fitting function. The standard Levenberg-Marquardt method for nonlinear fitting is used for the fitting. The rocking curve of the bandpass filter is included in the fitting function [13]. Figure 8 shows an example of the measured interferogram profile and the fitting function. In the case of Herschelian reflective optics, the value of $\gamma$ exceeded 0.9 and the measured vertical beam size was $4.73 \pm 0.55 \mu \mathrm{m}$ at a stored current of $1.5 \mathrm{~mA}$ under single-bunch operation. In the case of refractive optics, the value of $\gamma$ did not exceed 0.8 and the measured vertical beam size was $7.2 \pm 0.8 \mu \mathrm{m}$ under the same conditions. The effectiveness of Herschelian reflective optics was therefore confirmed.

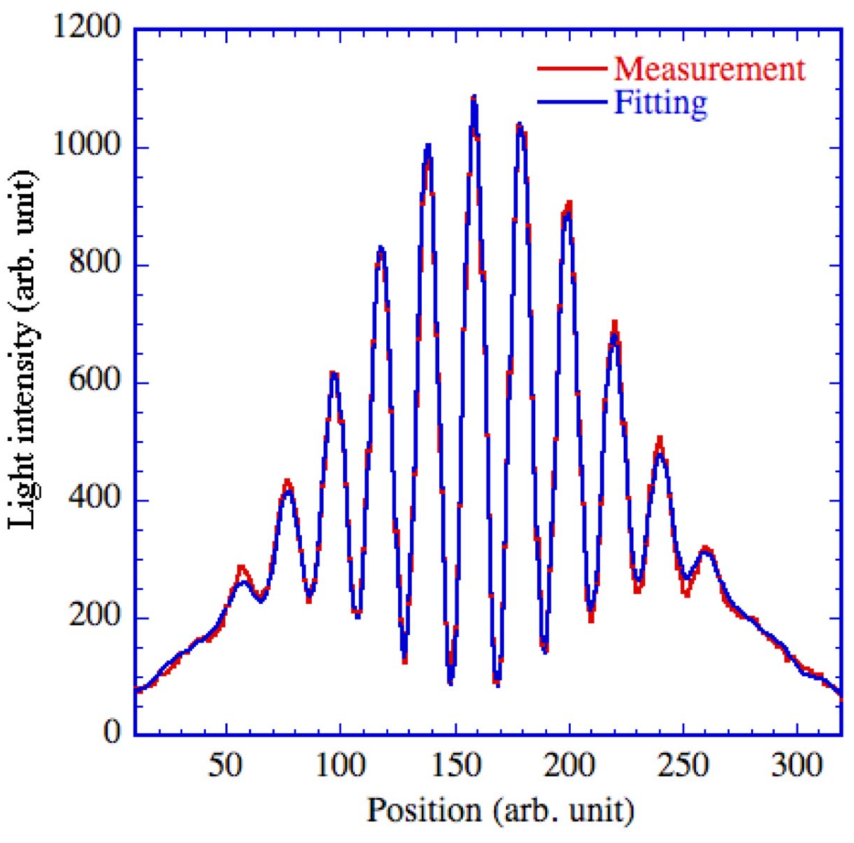

FIG. 8. (Color) Measured interferogram and fitting function.

\section{EMITTANCE TUNING OF THE DR}

The measurement strongly depends on the emittance tuning and the stored current in the DR. The vertical emittance $\varepsilon$ is estimated using the following equation:

$$
\varepsilon \beta=\sigma^{2}-\left[\eta\left(\sigma_{p} / p\right)\right]^{2},
$$

where $\beta$ denotes the beta function at the source point, $\eta$ denotes the dispersion function, and $\sigma_{p} / p$ denotes the momentum spread of the beam. The beta function at the quadrupole magnets near the source point is measured from the quadrupole strength dependence of the betatron tune. The beta function at the source point is estimated from extrapolation of the fitting of the beta functions between two quadrupole magnets, which are set downstream and upstream of the source point. The value of the dispersion function at the source point is negligible for the vertical direction.

A low-vertical-emittance tuning method was developed $[10,11]$. Some of the corrections are sequentially iterated to minimize the emittance; namely, the closed orbit distortion correction, vertical dispersion correction, and $x-y$ coupling correction. Because the vertical emittance of the DR is strongly dependent on the vertical dispersion and the $x-y$ coupling, these corrections are essential. The $x-y$ coupling is controlled by the strength of the skew component of each sextupole magnet (SF, SD).

\section{RESULTS OF EMITTANCE MEASUREMENT}

Vertical beam-size measurement using the SR interferometer with Herschelian reflective optics was performed with a stored current of 1-6 mA at a single bunch and an 
accelerating voltage of $300 \mathrm{kV}$. In order to change the $x-y$ coupling by controlling the vertical emittance, the on and off conditions of the skew coils of the sextupole magnets were measured. We defined the condition of both the SF and the SD skew coils being on as SQ1, the condition of the SF skew coils being on and the SD skew coils off as SQ2,

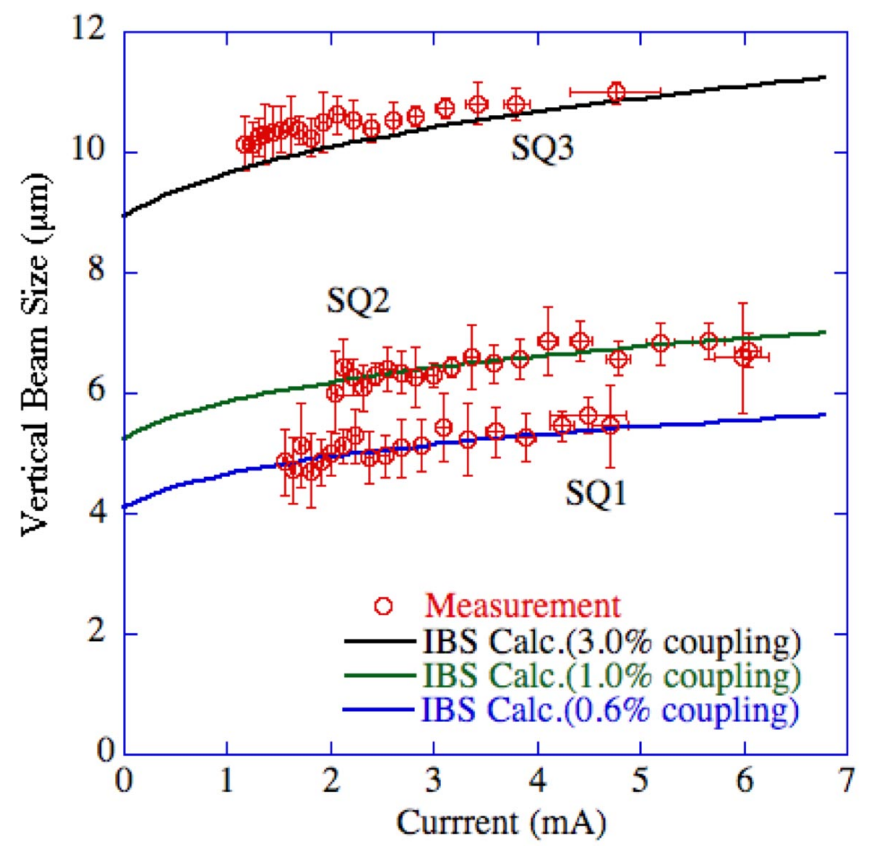

(a)

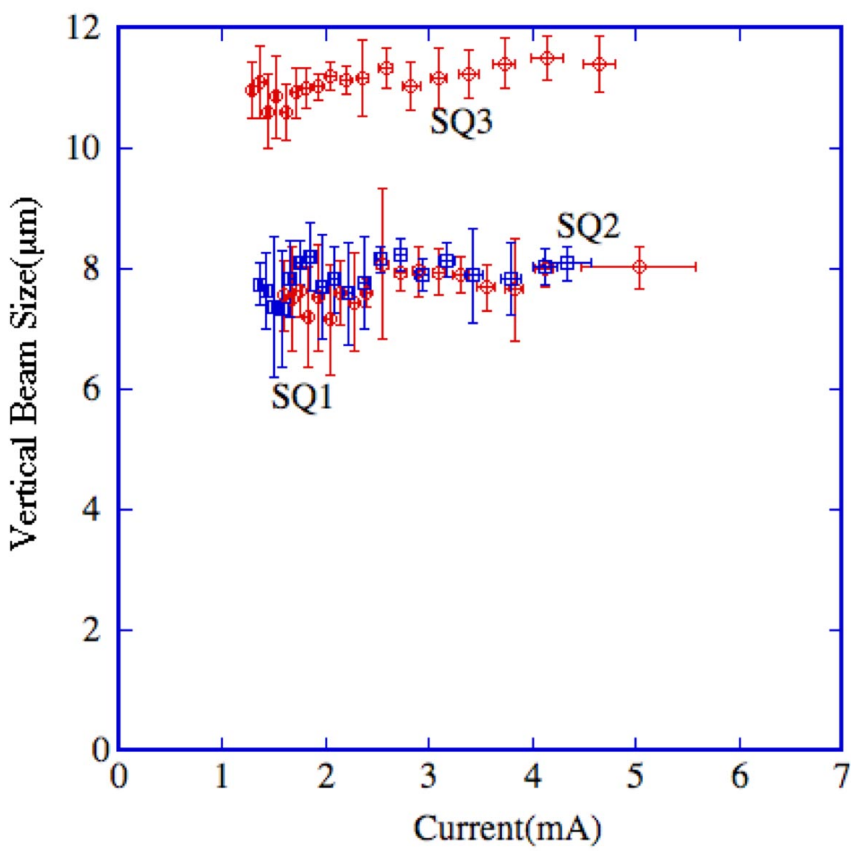

(b)

FIG. 9. (Color) (a) Vertical beam size as a function of the stored current measured with Herschelian reflective optics. (b) Vertical beam size as a function of the stored current measured with refractive optics. and the condition of both the SF and the SD skew coils being off as SQ3. The measured vertical beam size as a function of the stored current is shown in Fig. 9(a). In the case of the SQ1 condition, the measured vertical size was $4.7 \mu \mathrm{m}$ at a stored current of $1 \mathrm{~mA}$, which corresponds to a vertical emittance of $0.97 \times 10^{-11} \mathrm{~m}$. The measured vertical beta function $(\beta y=2.35 \mathrm{~m})$ was used for the calculation.

The intensity dependence of the vertical beam size is explained by the intrabeam scattering (IBS) effect. The IBS effect is computed using the SAD program [14]. The measurements of SQ1, SQ2, and SQ3 showed good agreement with the calculations in the case of $x-y$ couplings of $0.6 \%, 1.0 \%$, and $3.0 \%$, respectively. The calculation used a bunch length of $5.2 \mathrm{~mm}$ and an energy spread of $6 \times 10^{-4}$ at zero current.

The same measurement by refractive optics, using an achromatic lens with a focal length of $600 \mathrm{~mm}$ and a diameter of $80 \mathrm{~mm}$ for the objective, is shown in Fig. 9(b) for comparison. A bandpass filter (450 nm with $80 \mathrm{~nm}$ bandwidth) was used. In the case of the SQ3 condition, the measurement closely agreed with the results of the Herschelian reflective optics; however, the measurements of the SQ2 and the SQ1 conditions showed a clear discrepancy from the results of the Herschelian reflective optics, with a somewhat lower limit of measurement of around $7 \mu \mathrm{m}$.

\section{CONCLUSION}

The SR interferometer using refractive optics has a measurement limit of around $7 \mu \mathrm{m}$ in small beam-size measurement, which arises from the dispersion effect of the objective lens. The measurement error was quantitatively estimated by simulation. To eliminate the dispersion effect of the objective lens, Herschelian reflective optics was introduced to the SR interferometer. The SR interferometer with Herschelian reflective optics successfully measured a beam size of less than $5 \mu \mathrm{m}$ at the ATF-DR. An emittance study was carried out at the ATF-DR using the SR interferometer with Herschelian reflective optics. The measured vertical beam size agreed well with the IBS calculation at each $x-y$ coupling ratio. The measured $x-y$ coupling ratio was $0.6 \%$ and the measured emittance was $0.97 \times 10^{-11} \mathrm{~m}$ at a storage current of $1 \mathrm{~mA}$ under singlebunch operation.

The effectiveness of the Herschelian reflective optics of the SR interferometer was confirmed by a comparison with the refractive optics.

\section{ACKNOWLEDGMENTS}

The authors would like to express their gratitude to Professor Y. Kamiya and Professor K. Yokoya for their encouragement. They also wish to thank Professor J. Urakawa for his leadership of the ATF project, and the 
ATF staff for their support during the measurements.

[1] ILC baseline configuration document, 2006: http:// www.linearcollider.org/

[2] F. Hinode et al., KEK Internal Report 95-4, 1995.

[3] T. Mitsuhashi, Proceedings of the Joint US-CERN-JapanRussia School on Particle Accelerator Beam Measurements (World Scientific Publishing, Singapore, 1999), pp. 399-427.

[4] J. W. Flanagan et al., Proceedings of EPAC 2000, Vienna, 2000, p. 1714.

[5] A.S. Fisher et al., Proceedings of PAC 2001, Chicago, 2001, p. 547.
[6] I. Sakai et al., Rev. Sci. Instrum. 71, 1264 (2000).

[7] M. Masaki et al., J. Synchrotron Radiat. 10, 295 (2003).

[8] T. Mitsuhashi et al., Proceedings of the 6th European Particle Accelerator Conference, Stockholm, 1998.

[9] T. Naito et al., Proceedings of the Particle Accelerator Conference, New York, 1999.

[10] K. Kubo et al., Proceedings of the European Particle Accelerator Conference, Vienna, 2000, p. 483.

[11] Y. Honda et al., Phys. Rev. Lett. 92, 054802 (2004).

[12] M. Born and E. Wolf, Principles of Optics (Pergamon Press Ltd., New York, 1980).

[13] J. W. Flanagan et al., Proceedings of the 12th Symposium on Accelerator Science and Technology, 1999.

[14] SAD is a computer program complex for accelerator design: http://www-acc-theory.kek.jp/SAD/sad.html 